\title{
Crizotinib versus Alectinib for the Treatment of ALK-Positive Non-Small Cell Lung Cancer: A Systematic Review and Meta-Analysis
}

\author{
Qinghua Zeng $^{\mathrm{a}} \quad$ Xiquan Zhang $^{\mathrm{b}}$ Shan He ${ }^{\mathrm{a}}$ Zhiyong Zhou ${ }^{\mathrm{b}}$ Luping Xia $^{\mathrm{a}}$ \\ Wenxiong Zhang ${ }^{c}$ Lin Zeng ${ }^{b}$ \\ aDepartment of Pulmonary and Critical Care Medicine, The First Affiliated Hospital of Nanchang University, \\ Nanchang, PR China; 'D Department of Oncology, Jiangxi Provincial People's Hospital, Nanchang, PR China; \\ 'Department of Thoracic Surgery, The Second Affiliated Hospital of Nanchang University, Nanchang, PR China
}

\section{Keywords}

Crizotinib · Alectinib · Anaplastic lymphoma kinase ·

Non-small cell lung cancer $\cdot$ Meta-analysis

\begin{abstract}
Background: Crizotinib and alectinib are the 2 most commonly used anaplastic lymphoma kinase (ALK) inhibitors for ALK-positive non-small cell lung cancer (NSCLC). We compared their antitumor efficacies and adverse effects based on a pooled analysis of the ALEX, ALESIA, and J-ALEX clinical trials. Methods: Seven databases were searched for eligible articles. The primary endpoints included overall survival (OS), progression-free survival (PFS), central nervous system (CNS)-PFS, drug responses, and adverse effects (AEs). $\boldsymbol{R e}$ sults: Seven articles on 3 randomized controlled clinical trials (ALEX, ALESIA, and J-ALEX) that included 697 patients were included. Compared with crizotinib, alectinib exhibited superior efficacy in PFS (HR [hazard ratio]: 0.35 [0.25-0.49], $p<$ 0.00001 ), OS (HR: 0.66 [0.47-0.92], $p=0.02$ ), CNS-PFS (HR: 0.17 [0.11-0.24], $p<0.00001$ ), duration of response (HR: 0.31 [0.23-0.42], $p<0.00001$ ), objective response rate (risk ratio [RR]: 0.87 [0.80-0.94], $p=0.0003$ ), partial response (RR: 0.88 [0.81-0.96], $p=0.004$ ), and grade 3-5 AEs (RR: 1.43 [1.09-
\end{abstract}

karger@karger.com

(c) 2021 S. Karger AG, Basel

www.karger.com/che

Karger $\stackrel{2}{5}$
1.87], $p=0.009$ ). Additionally, compared with crizotinib, alectinib exhibited a survival advantage that increased with its prolongation of survival time. The disease control rate, complete response, and total AEs were comparable between the 2 groups. The crizotinib group reported higher rates of constipation, nausea, diarrhea, vomiting, peripheral edema, dysgeusia, visual impairment, and levels of alanine aminotransferase and aspartate aminotransferase as well as greater decreases in appetite and neutrophil count. Conclusions: In both antitumor efficacy and safety, alectinib appears to be superior to crizotinib for the treatment of ALK-positive NSCLC.

(c) 2021 S. Karger AG, Basel

\section{Introduction}

Over the last decade, lung cancer has become the leading cause of cancer-related death, and $80 \%$ of all lung cancers are non-small cell lung cancer (NSCLC) [1]. Approximately $3 \%-7 \%$ of all patients with NSCLC have anaplastic lymphoma kinase (ALK)-positive disease [2]. Crizotinib, the first clinically established ALK inhibitor, exhibits satisfactory antitumor efficacy and safety com- 
pared with chemotherapy for treatment of ALK-positive NSCLC $[3,4]$. However, frequent crizotinib resistance and poor central nervous system (CNS) efficacy are very troubling for clinicians [5].

Alectinib, the most commonly used second-generation ALK inhibitor, is effective against several ALK mutations and exhibits high selectivity and CNS activity $[6,7]$. In the AF-001JP clinical trial, Seto et al. [8] demonstrated that alectinib is highly effective for the treatment of ALKpositive NSCLC. However, whether alectinib can replace crizotinib as the first-line treatment for patients with ALK-positive NSCLC remains controversial $[9,10]$. Peters et al. [11] reported that alectinib achieves better progression-free survival (PFS) and has fewer adverse effects (AEs) than crizotinib in treatment of ALK-positive NSCLC. Hida et al. [12] and Nishio et al. [13] suggested that alectinib could prevent new brain metastasis and avert the progression of brain metastases. In the ALESIA clinical trial, Zhou et al. [14] confirmed that alectinib treatment results in improved clinical benefits, e.g., PFS and CNS-PFS, for ALK-positive NSCLC. However, a statistically significant advantage of overall survival (OS) was still not confirmed in the ALEX, ALESIA, or J-ALEX trials [11, 12, 14]. Bedas et al. [15] reported that crizotinib and alectinib treatments were associated with similar PFS and OS rates in elderly patients with advanced ALK-positive NSCLC. To further clarify this debate, we compared the efficacy and safety of crizotinib with those of alectinib for treatment of ALK-positive NSCLC by performing a meta-analysis of the relevant literature.

\section{Materials and Methods}

We conducted this study according to the Preferred Reporting Items for Systematic Reviews and Meta-Analysis guidelines (online suppl. Table S1; for all online suppl. material, see www. karger.com/doi/10.1159/000521452) (Registration information: PROSPERO CRD42020185135).

\section{Search Strategy}

PubMed, EMBASE, Scopus, Ovid MEDLINE, Web of Science, the Cochrane Library, ScienceDirect, and Google Scholar were rigorously searched for eligible randomized, controlled trials (RCTs) from inception to March 5, 2021. As key words, we used "lung cancer," "crizotinib," and "alectinib." We also searched the reference lists of the included RCTs to identify additional eligible studies. Details can be found in online supplementary Table S2.

\section{Selection Criteria}

Studies that met the following criteria were enrolled in accordance with PICOS (Participants, Intervention, Control, Outcome, Study design):
1. Population (P): patients with ALK-positive NSCLC;

2. Intervention (I) and comparison (C): crizotinib versus alectinib;

3. Outcomes (O): antitumor efficacy and AEs (see the Data Extraction section);

4. Study design (S): RCTs published in English.

The following articles were excluded: articles without initial data, meta-analyses, conference articles, case reports, and articles from the same experimental center on the same topic. Different articles that focused on the same trial were included if they contained different outcomes; however, when analyzing the same outcome, only the most recent data were used.

\section{Data Extraction}

The following data were extracted by 2 independent investigators: the study characteristics (e.g., publication date, first author, and design), participant characteristics (e.g., number, sex, and age), cancer characteristics (e.g., histopathology, stage, and ALK status), antitumor efficacy (e.g., OS, PFS, CNS-PFS, and drug responses), and number of AEs (total AEs, grade 3-5 AEs, treatment discontinuation, dose reduction, and dose interruption). All data assessed by the independent review committee (IRC) and investigators were extracted. All disagreements between the 2 investigators were resolved through reexamination and discussion.

\section{Outcome Assessments}

OS, PFS, and CNS-PFS were the primary endpoints analyzed. In addition to analyzing the time-to-event data, we compared the rates of survival (OS rate [OSR], PFS rate [PFSR], and CNS-PFS rate [CNS-PFSR]) at $6,12,18,24$, and 30 months (OSR 6-30 months, PFSR 6-30 months, and CNS-PFSR 6-30 months) between the 2 groups. Additionally, we analyzed PFS according to the following subgroups: age, sex, smoking status, CNS metastases at baseline, race category, treatment line, previous brain radiation, disease stage, and the ALK testing method.

\section{Quality Assessment}

The quality of RCTs was assessed using the 5-point Jadad scale and the Cochrane Risk Assessment Tool. The Jadad scale is primarily used to evaluate quality based on randomization, blinding, and patient inclusion. A study is regarded as high quality if it receives a score of $\geq 3$ points [16]. The Cochrane Risk Assessment Tool primarily focuses on the bias of selection, performance, detection, attrition, and reporting, and the risk is assessed as low, unclear, or high risk [17]. Then, the results are presented as a risk of bias graph.

The quality of the results was assessed using the Grading of Recommendations, Assessment, Development, and Evaluation (GRADE) method [18]. The GRADE approach primarily focuses on bias, discordance, indirectness, inaccuracy, and publication bias. The results include 4 levels: very low, low, medium, and high.

\section{Statistical Analysis}

We used Review Manager 5.3 software (Nordic Cochrane Center, Oxford, UK) to evaluate the pooled data. The hazard ratio (HR) was used to analyze the survival data (OS, PFS, and CNSPFS). All of the HR data were directly extracted from the included studies. When the HR was $<1$, the results supported the alectinib group. The risk ratio (RR) was used to analyze the dichotomous variables (drug responses, OSR, PFSR, CNS-PFSR, and AEs). 


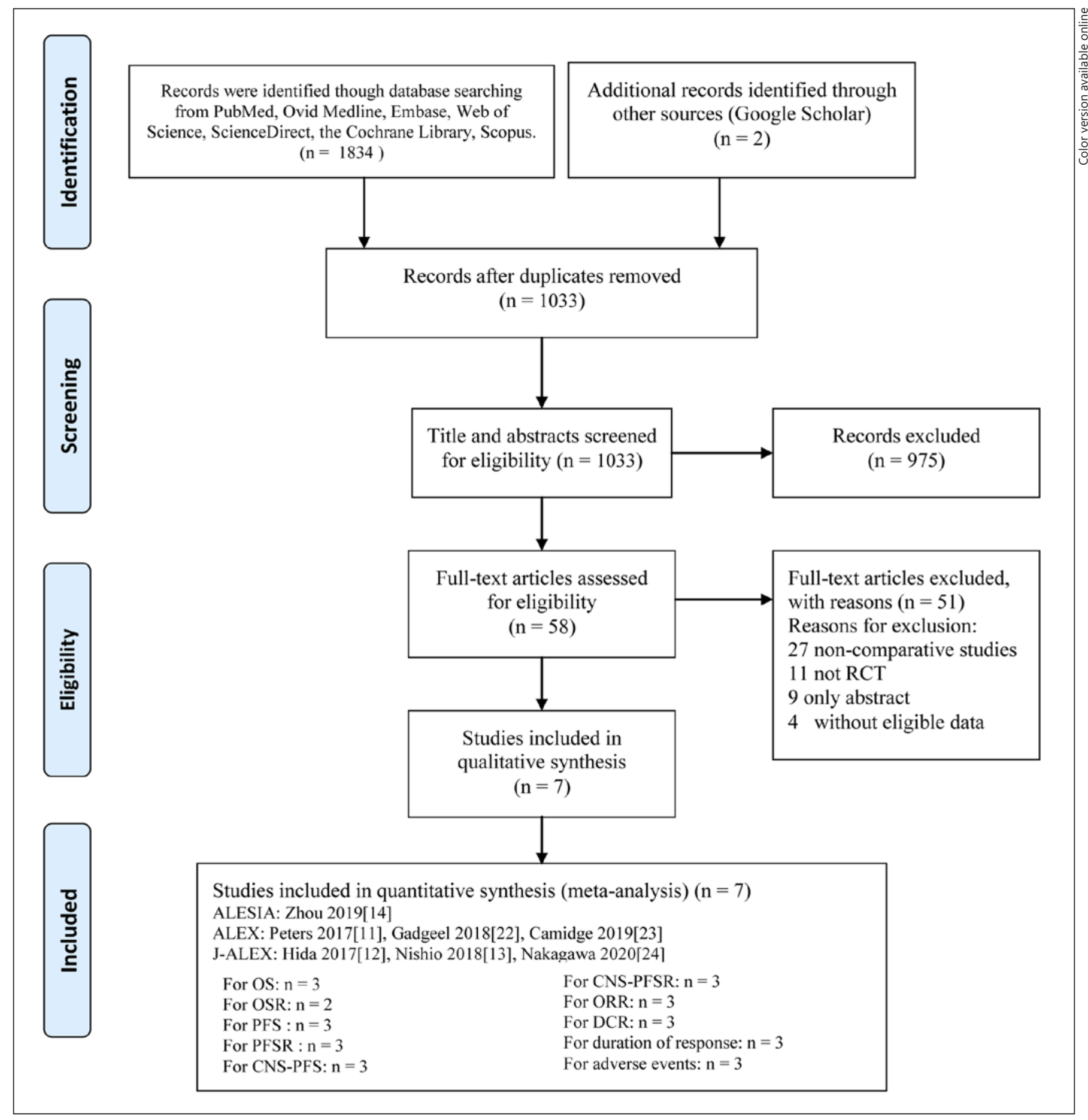

Fig. 1. Flowchart of study selection.

When the RR was $>1$, then the results supported the alectinib group, as in the analysis of AEs, or the results supported the crizotinib group, as in the analysis of OSR, PFSR, CNS-PFSR, and AEs. The antitumor efficacy data assessed by the IRC and investigators were analyzed separately. We used the $I^{2}$ statistic and $\chi^{2}$ test to evaluate the heterogeneity. If $I^{2}<50 \%$ or $p>0.1$, indicating no significant heterogeneity, then we used a fixed-effects model; otherwise, we used a random-effects model. Statistical significance was indicated by $p<0.05$. Publication bias was assessed by performing a visual check of the funnel plots. 
Table 1. Characteristics of the 3 randomized controlled trials (ALEX, ALESIA, and J-ALEX)

\begin{tabular}{|c|c|c|c|c|c|c|}
\hline Register No. & \multicolumn{2}{|c|}{ NCT02838420 } & \multicolumn{2}{|c|}{ NCT02075840 } & \multicolumn{2}{|c|}{ JapicCTI-132316 } \\
\hline Clinical trial stage & \multicolumn{2}{|l|}{ Phase III } & \multicolumn{2}{|l|}{ Phase III } & \multicolumn{2}{|l|}{ Phase III } \\
\hline Treatment line & \multicolumn{2}{|l|}{1} & \multicolumn{2}{|l|}{1} & \multicolumn{2}{|l|}{1 or 2} \\
\hline Country & \multicolumn{2}{|c|}{ China, South Korea, and Thailand } & \multicolumn{2}{|c|}{ Multicountries $^{\mathrm{a}}$} & \multicolumn{2}{|l|}{ Japan } \\
\hline Period & \multicolumn{2}{|c|}{ Aug 2016-May 2017} & \multicolumn{2}{|c|}{ Aug 2014-Jan 2016} & \multicolumn{2}{|c|}{ Nov 2013-Aug 2015} \\
\hline Treatment arms & Crizotinib & Alectinib & Crizotinib & Alectinib & Crizotinib & Alectinib \\
\hline Median age, years & 49 & 51 & 54 & 58 & 59.5 & 61 \\
\hline \multicolumn{7}{|l|}{ Race } \\
\hline Asian & 62 & 125 & 69 & 69 & 104 & 103 \\
\hline Non-Asian & 0 & 0 & 82 & 83 & 0 & 0 \\
\hline Median duration of therapy, months & 12.6 & 14.7 & 10.7 & 17.9 & - & - \\
\hline ECOG status & $0-2$ & & $0-2$ & & $0-2$ & \\
\hline Follow-up duration, months & 15 & 16.2 & 22.8 & 27.8 & 42.2 & 42.4 \\
\hline Pathology & \multicolumn{2}{|c|}{ ALK-positive NSCLC } & \multicolumn{2}{|c|}{ ALK-positive NSCLC } & \multicolumn{2}{|c|}{ ALK-positive NSCLC } \\
\hline Brain metastasis & & & & & & \\
\hline Measurable & 7 & 17 & 22 & 21 & 29 & 15 \\
\hline Nonmeasurable & 16 & 27 & 36 & 43 & & \\
\hline No & 39 & 81 & 93 & 88 & 75 & 89 \\
\hline Tumor response assessment & RECIST, ver & & RECIST, ver & & RECIST, ver & \\
\hline Brain metastasis assessment & MRI in all $p$ & & MRI in all $p$ & & MRI in all $p$ & \\
\hline Adverse events assessment & $\mathrm{NCl}-\mathrm{CTCAE}$ & 4.0 & $\mathrm{NCl}-\mathrm{CTCAE}$ & 4.0 & $\mathrm{NCl}-\mathrm{CTCAE}$ & \\
\hline Frequency of disease assessment & Every 8 we & I DP & Every 8 we & DP & Every 4 we & veek 12 \\
\hline & & & & & $\begin{array}{l}\text { Every } 8 \text { we } \\
\text { week } 76\end{array}$ & veek 12 to \\
\hline & & & & & Every $12 \mathrm{w}$ & after until DP \\
\hline Funding & F. Hoffman & che, Ltd. & F. Hoffman & he, Ltd. & Chugai Pha & cal Co., Ltd. \\
\hline
\end{tabular}

$\mathrm{RCT}$, randomized controlled trial; NSCLC, non-small cell lung cancer; bid, twice a day; ECOG, Eastern Cooperative Oncology Group; M/F, male/female; ALK, anaplastic lymphoma kinase; NCl-CTCAE, National Cancer Institute Common Terminology Criteria for Adverse; IHC, immunohistochemistry; FISH, fluorescence in situ hybridization; MRI, magnetic resonance imaging; RECIST, Response Evaluation Criteria In Solid Tumors; RT-PCR, reverse transcription-polymerase chain reaction. ${ }^{a}$ Multicountries including Australia, Bosnia and Herzegovina, Brazil, Canada, Chile, China, Costa Rica, Denmark, Dominican Republic, Egypt, France, Germany, Greece, Guatemala, Hong Kong, Israel, Italy, Mexico, New Zealand, Peru, Poland, Portugal, Republic of Korea, Russia, Serbia, Singapore, Spain, Switzerland, Taiwan, Thailand, Turkey, Ukraine, UK, and USA. 


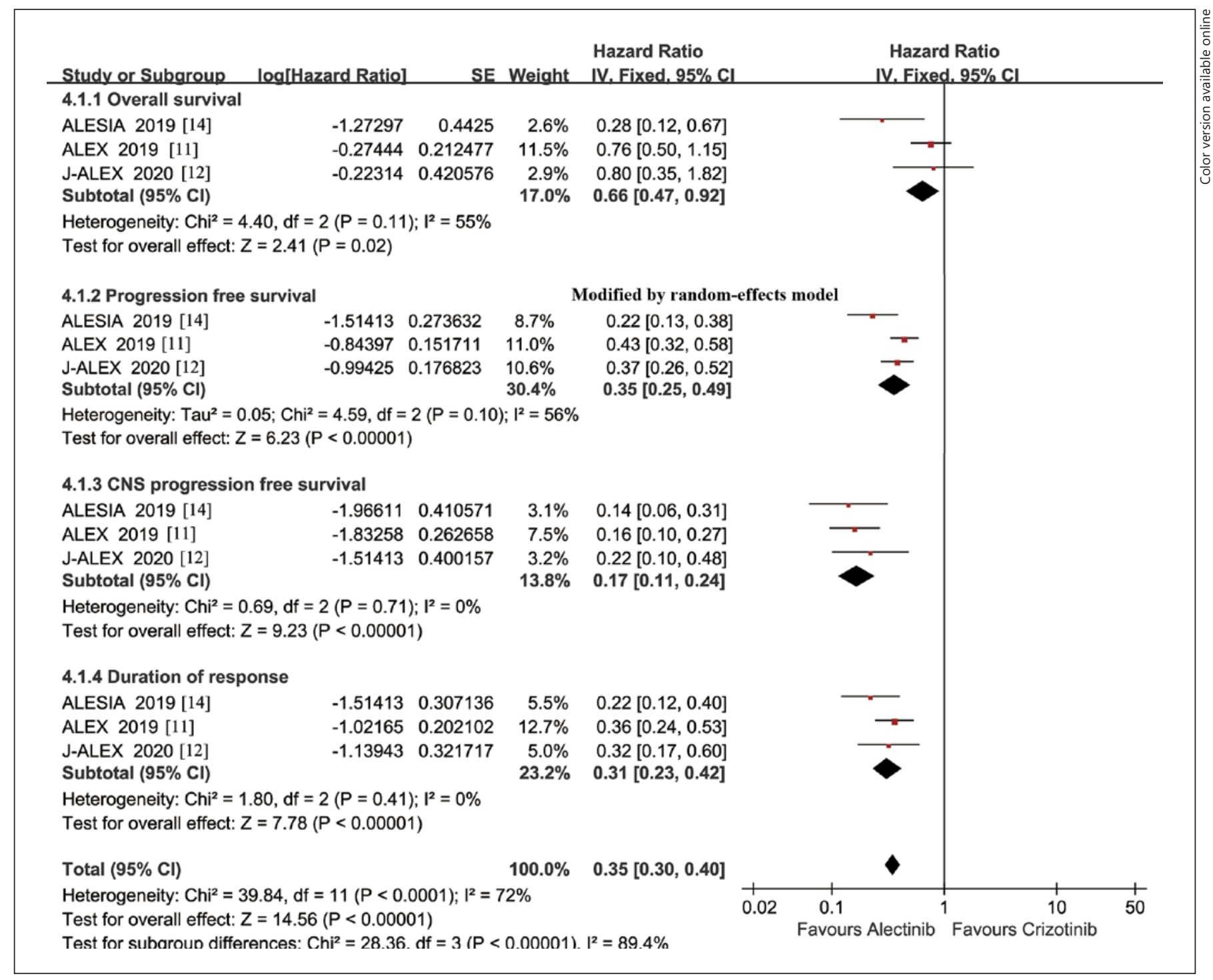

Fig. 2. Forest plots of OS, PFS, CNS-PFS, and DOR associated with crizotinib versus alectinib.

\section{Results}

\section{Search Results}

Seven articles on 3 RCTs (ALEX, ALESIA, and J-ALEX) that included 697 patients (317 patients in the crizotinib arm and 380 patients in the alectinib arm) were included in the final analysis [11-14, 19-21] (Fig. 1). The ALESIA and J-ALEX trials $[12,14]$ were conducted in Asia, but the ALEX trial included participants from 32 countries all over the world [11]. All 3 of these studies were of high quality according to the Cochrane Risk of Bias Tool (online suppl. Fig. S1) and the Jadad scale (online suppl. Table S3). According to the GRADE method, all of the results were of medium-high quality (online suppl. Table S4). Essential information from the ALEX, ALESIA, and J-ALEX clinical trials is summarized in Table 1.

\section{Antitumor Efficacy}

Three studies compared the OS of the alectinib group versus the crizotinib group (heterogeneity: $I^{2}=55 \%, p=$ $0.02)$. The OS of the alectinib group was better than that of the crizotinib group (HR: $0.66,95 \%$ confidence interval [CI]: [0.47-0.92], $p=0.02$; Fig. 2). The OSR at all time points tended to favor the alectinib group but was not significantly different between the groups (OSR-6 

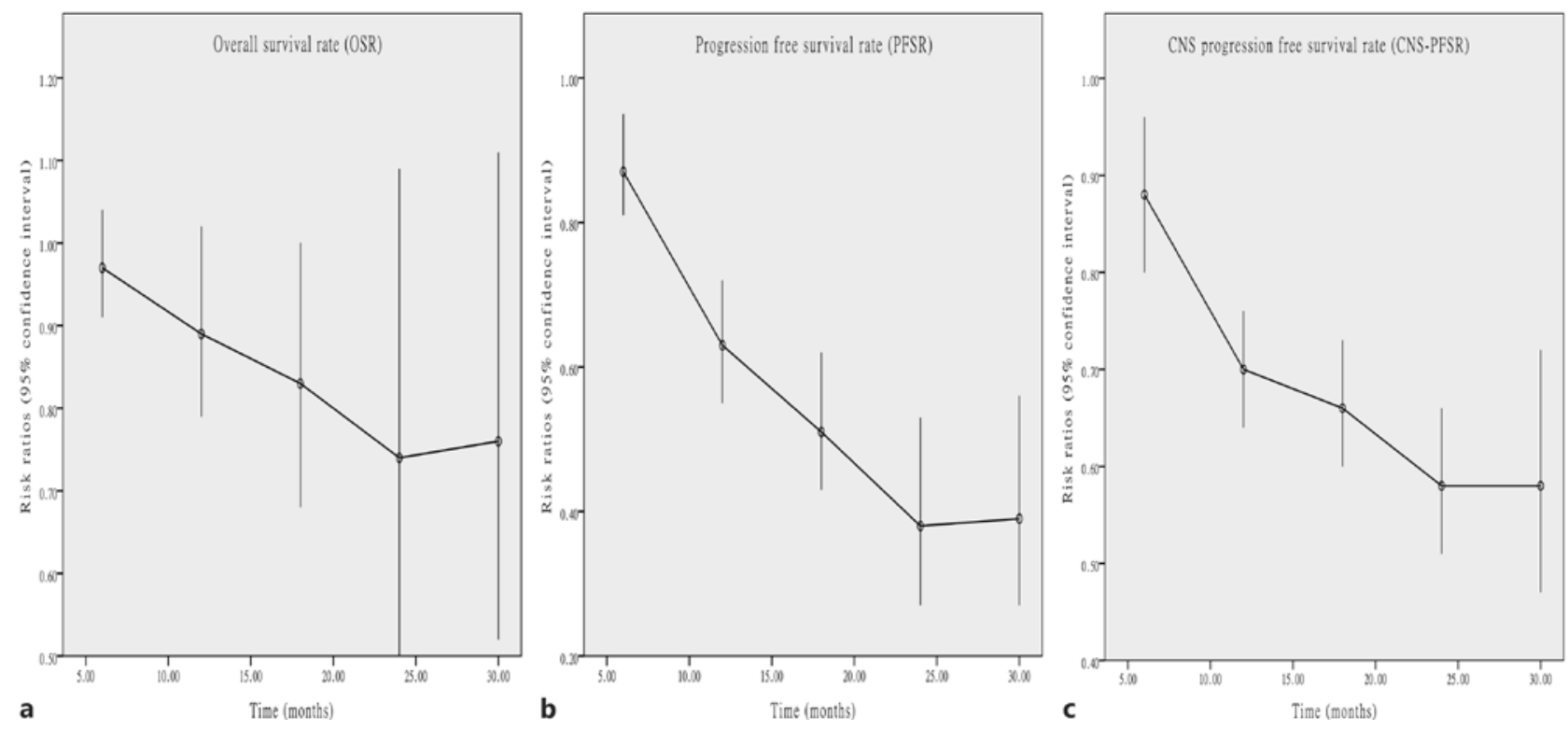

Fig. 3. Line charts of OSR (6-30 months; a), PFSR (6-30 months; b), and CNS-PFSR (6-30 months; c) associated with crizotinib versus alectinib according to survival time.

months, RR: 0.97 [0.91-1.04], $p=0.36$; OSR-12 months, RR: 0.89 [0.79-1.02], $p=0.09$; OSR-18 months, RR: 0.83 [0.68-1.00], $p=0.05$; OSR-24 months, RR: 0.74 [0.50$1.09], p=0.13$; and OSR-30 months, RR: 0.76 [0.52-1.11], $p=0.16$; online suppl. Fig. S2). With prolonged survival, alectinib exhibited an increased advantage in OS compared with crizotinib (Fig. 3a; online suppl. Fig. S3a).

Three studies compared the PFS of the alectinib group versus the crizotinib group (heterogeneity: $I^{2}=56 \%, p=$ $0.10)$. The PFS of the alectinib group was better than that of the crizotinib group (HR: 0.35 [0.25-0.49], $p<0.00001$; Fig. 2). The PFSR at all time points significantly favored the alectinib group (PFSR-6 months, RR: 0.87 [0.810.95], $p=0.0009$; PFSR-12 months, RR: 0.63 [0.55-0.72], $p<0.00001$; PFSR-18 months, RR: 0.51 [0.43-0.62], $p<$ $0.00001 ;$ PFSR-24 months, RR: $0.38[0.27-0.53], p<$ 0.00001; and PFSR-30 months, RR: 0.39 [0.27-0.56], $p<$ 0.00001; online suppl. Fig. S4). With prolonged survival, alectinib showed an increased advantage in PFS compared with crizotinib (Fig. 3b; online suppl. Fig. S3b). In the subgroup analysis, significant changes in PFS were not observed according to age, sex, race category, or treatment line. Smoking status (active smoker), Eastern Cooperative Oncology Group-performance status (ECOG-PS) 2 , disease stage (postoperative recurrence), and the ALK testing method (reverse transcription-polymerase chain reaction [RT-PCR]) might be unfavorable factors for alectinib treatment. However, baseline CNS metastases and previous brain radiation might be favorable factors for alectinib treatment (Fig. 4).

Three studies compared the CNS-PFS of the alectinib group versus the crizotinib group (heterogeneity: $I^{2}=0 \%$, $p=0.71$ ). The CNS-PFS of the alectinib group was better than that of the crizotinib group (HR: 0.17 [0.11-0.24], $p<0.00001$; Fig. 2). The CNS-PFSR at all time points significantly favored the alectinib group (CNS-PFSR-6 months, RR: 0.88 [0.80-0.96], $p=0.005$; CNS-PFSR-12 months, RR: 0.70 [0.64-0.76], $p<0.00001$; CNS-PFSR-18 months, RR: 0.66 [0.60-0.73], $p<0.00001$; CNS-PFSR-24 months, RR: 0.58 [0.51-0.66], $p<0.00001$; and CNSPFSR-30 months, RR: 0.58 [0.47-0.72], $p<0.00001$; online suppl. Fig. S5). With prolonged survival, alectinib exhibited an increased advantage in CNS-PFS compared with crizotinib (Fig. 3c; online suppl. Fig. S3c).

Three studies compared the duration of response (DOR) of the alectinib group versus the crizotinib group (heterogeneity: $I^{2}=0 \%, p=0.41$ ). The DOR of the alectinib group was better than that of the crizotinib group (HR: 0.31 [0.23-0.42], $p<0.00001$; Fig. 2). The objective response rate (ORR) (74.45\% vs. $86.58 \%$, RR: 0.87 [0.80- 


\begin{tabular}{|c|c|c|c|c|c|c|c|c|c|}
\hline \multirow{2}{*}{ Subgroups } & \multirow{2}{*}{$\begin{array}{l}\text { Included } \\
\text { studies }\end{array}$} & \multirow{2}{*}{ Total } & \multicolumn{2}{|c|}{ Crizotinib } & \multicolumn{2}{|c|}{ Alectinib } & & & \multirow{2}{*}{ HR ( $95 \%$ CI) } \\
\hline & & & Events & $\mathrm{n}$ & Events & $\mathrm{n}$ & & & \\
\hline All patients & 3 & 697 & 211 & 317 & 123 & 380 & 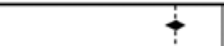 & & $0.35(0.25,0.49)$ \\
\hline \multicolumn{10}{|l|}{ Age } \\
\hline$<65$ years & 3 & 584 & 173 & 264 & 102 & 320 & - & & $0.37(0.29,0.47)$ \\
\hline$>65$ years & 3 & 113 & 38 & 53 & 21 & 60 & & & $0.35(0.20,0.62)$ \\
\hline \multicolumn{10}{|l|}{ Sex } \\
\hline Female & 3 & 385 & 114 & 178 & 65 & 207 & $\rightarrow$ & & $0.35(0.26,0.49)$ \\
\hline Male & 3 & 312 & 97 & 139 & 58 & 173 & & & $0.32(0.16,0.64)$ \\
\hline \multicolumn{10}{|l|}{ Smoking status } \\
\hline Active smoker & 3 & 114 & 32 & 51 & 15 & 63 & & & $0.42(0.08,2.24)$ \\
\hline Former smoker & 2 & 147 & 47 & 62 & 28 & 85 & & & $0.27(0.10,0.69)$ \\
\hline Non-smoker & 3 & 436 & 132 & 184 & 80 & 232 & > & & $0.40(0.30,0.53)$ \\
\hline \multicolumn{10}{|l|}{ ECOG PS } \\
\hline 0 & 3 & 326 & 98 & 164 & 46 & 162 & & & $0.36(0.25,0.52)$ \\
\hline 1 & 2 & 342 & 102 & 140 & 67 & 202 & & & $0.30(0.15,0.62)$ \\
\hline 2 & 3 & 29 & 11 & 13 & 10 & 16 & & & $0.79(0.29,2.16)$ \\
\hline \multicolumn{10}{|l|}{ CNS metastases at baseline } \\
\hline Yes & 3 & 232 & 84 & 110 & 42 & 122 & & & $0.18(0.07,0.48)$ \\
\hline No & 3 & 465 & 127 & 207 & 81 & 258 & & & $0.42(0.31,0.55)$ \\
\hline \multicolumn{10}{|l|}{ Previous brain radiation } \\
\hline Yes & 2 & 60 & 23 & 26 & 14 & 34 & & & $0.23(0.11,0.49)$ \\
\hline No & 2 & 430 & 130 & 187 & 84 & 243 & & & $0.36(0.19,0.66)$ \\
\hline \multicolumn{10}{|l|}{ Race category } \\
\hline Asian & 3 & 532 & 146 & 235 & 83 & 297 & 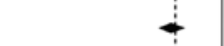 & & $0.34(0.26,0.44)$ \\
\hline Non-asian & 1 & 165 & 65 & 82 & 40 & 83 & & & $0.44(0.30,0.65)$ \\
\hline \multicolumn{10}{|l|}{ Treatment line } \\
\hline First line & 3 & 623 & 188 & 280 & 113 & 343 & & & $0.36(0.28,0.46)$ \\
\hline Second line & 1 & 74 & 23 & 37 & 10 & 37 & & & $0.39(0.18,0.84)$ \\
\hline \multicolumn{10}{|l|}{ Disease stage } \\
\hline Stage IIIB or IV & 3 & 647 & 198 & 291 & 117 & 356 & : & & $0.36(0.28,0.45)$ \\
\hline Postoperative recurrence & 1 & 50 & 13 & 26 & 6 & 24 & & & $0.49(0.18,1.32)$ \\
\hline \multicolumn{10}{|l|}{ ALK testing method } \\
\hline Single confirmation (IHC or RT-PCR) & 3 & 507 & 159 & 223 & 102 & 284 & - & & $0.38(0.30,0.50)$ \\
\hline \multirow[t]{2}{*}{ Double confirmation (IHC and FISH) } & 1 & 190 & 52 & 94 & 21 & 96 & & & $0.30(0.18,0.50)$ \\
\hline & & & & & & & $\begin{array}{l}0.1 \\
0.01 \\
\text { Favours Alectinib }\end{array}$ & ${ }_{\text {Favours Crizotinib }}^{10}$ & \\
\hline
\end{tabular}

Fig. 4. Forest plots of PFS in patient subgroups.

$0.94], p=0.0003)$ and the partial response [PR] $(71.92 \%$ vs. $82.11 \%$, RR: 0.88 [0.81-0.96], $p=0.004)$ were better in the alectinib group. The disease control rate [DCR] (90.54\% vs. 94.21\%, RR: 0.95 [0.88-1.04], $p=0.27$ ) and the complete response [CR] $(2.52 \%$ vs. $4.47 \%$, RR: 0.57 [0.26-1.32], $p=0.20$ ) were similar between the groups. Due to the high ORR and similar DCR in the alectinib

Crizotinib versus Alectinib for ALK-

Positive NSCLC group, less stable disease [SD] (16.09\% vs. 7.63\%, RR: 2.01 [13.1-3.10], $p=0.001$ ) was found in the alectinib group (Fig. 5).

Subgroup analysis was conducted according to whether patients had baseline CNS metastases. In patients with CNS lesions at baseline, alectinib was superior to crizotinib in PFS (HR: 0.23 [0.11-0.49], $p=0.0001$ ), systemic 


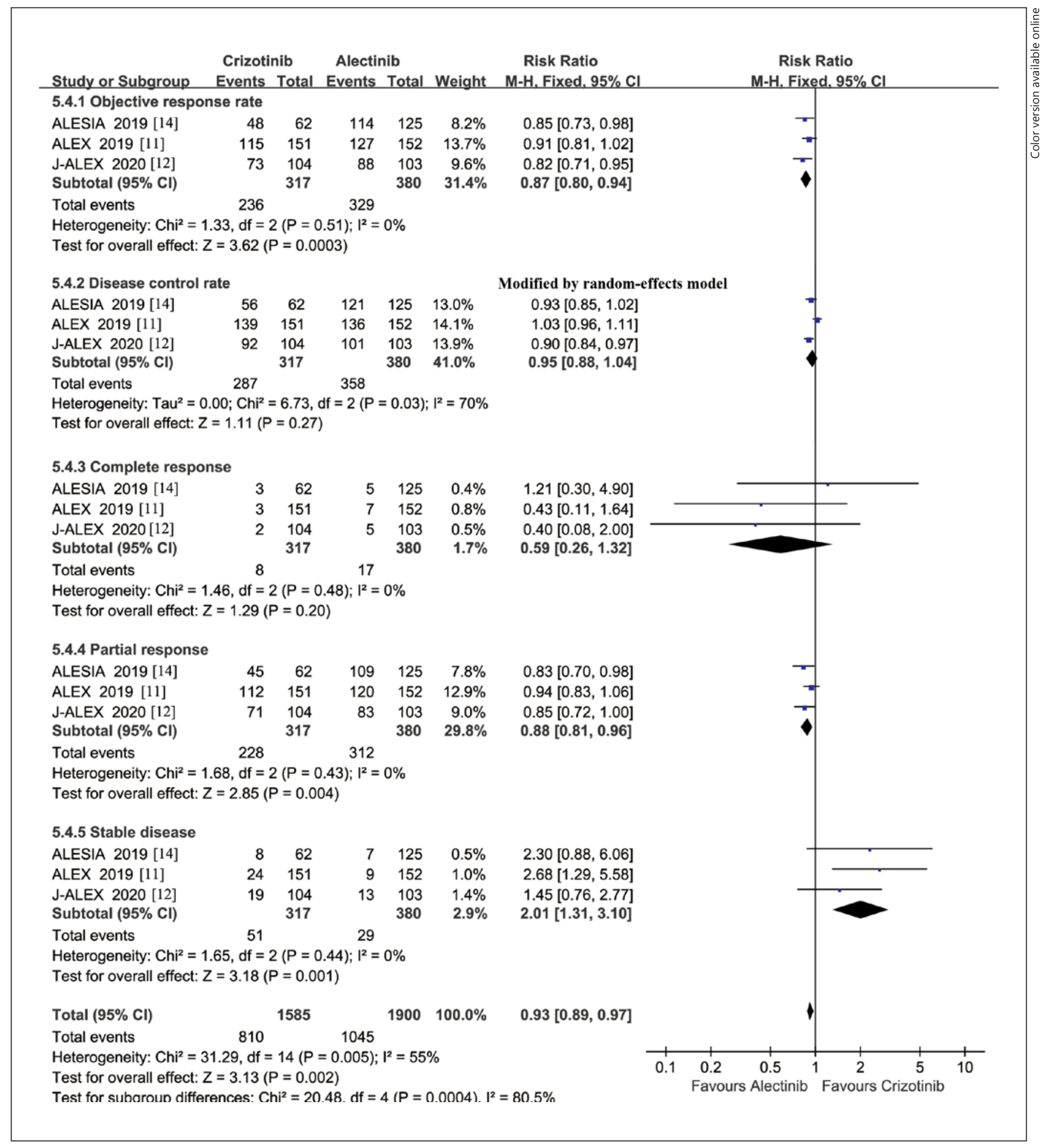

Fig. 5. Forest plots of drug responses (ORR, DCR, CR, PR, and SD) associated with crizotinib versus alectinib. 
Table 2. Summary of adverse events

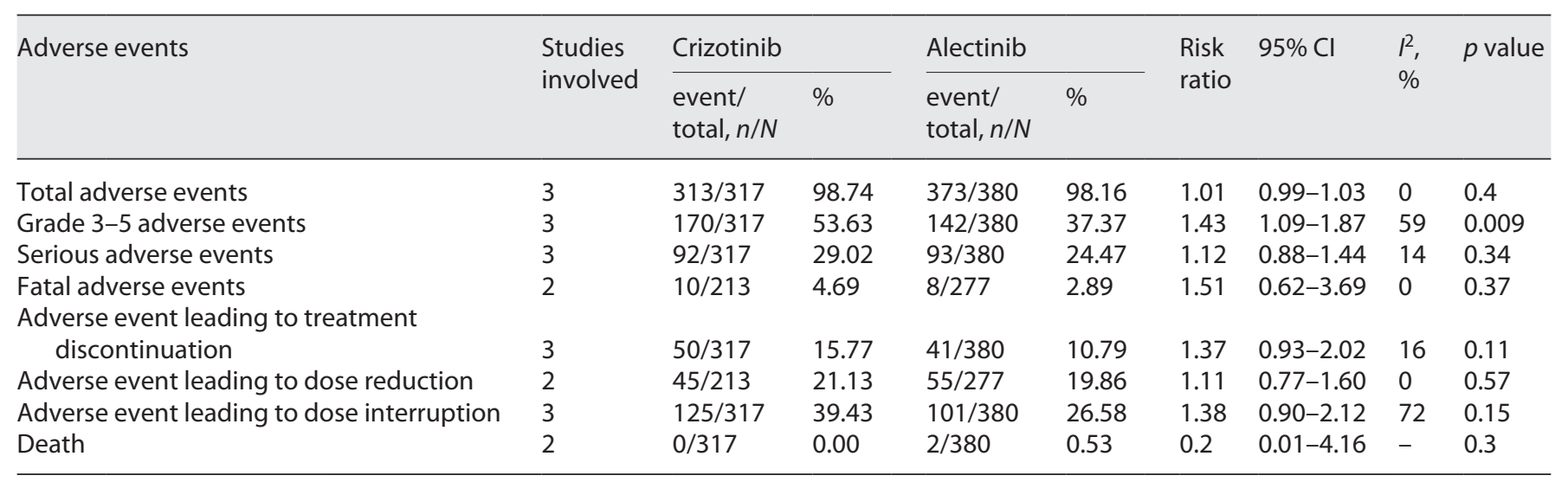

$\mathrm{Cl}$, confidence interval.

progression without previous CNS disease progression (HR: 0.35 [0.15-0.83], $p=0.02$ ), and CNS progression without previous systemic disease progression (HR: 0.18 $[0.09-0.36], p<0.00001)$. In patients without CNS lesions at baseline, the alectinib group also had better PFS (HR: $0.43[0.30-0.60], p<0.00001)$ and CNS progression without previous systemic disease progression (HR: 0.14 $[0.06-0.33], p<0.00001)$ than the crizotinib group. Similar mortality without previous CNS or systemic disease progression was found between patients with or without $\mathrm{CNS}$ lesions at baseline in the 2 groups (online suppl. Table S5).

All 3 studies reported data on survival and drug responses based on both IRC assessment and investigator assessment. According to the assessment of the IRC, the alectinib group had better OS (HR: 0.66 [0.47-0.92], $p=$ 0.02), PFS (HR: 0.42 [0.34-0.52], $p<0.00001$ ), CNS-PFS (HR: 0.18 [0.11-0.27], $p<0.00001$ ), and DOR (HR: 0.32 $[0.17-0.60], p=0.0004)$ than the crizotinib group. According to the assessment of the investigators, the alectinib group also had better OS (HR: 0.66 [0.47-0.92], $p=$ 0.02), PFS (HR: 0.35 [0.25-0.49], $p<0.00001$ ), CNS-PFS (HR: 0.14 [0.06-0.31],$p<0.00001$ ), and DOR (HR: 0.31 [0.22-0.43], $p<0.00001$ ) than the crizotinib group (online suppl. Table S6). Online supplementary Table S6 shows the results of responses and CNS responses assessed by the IRC and investigators.

\section{Toxicity}

In summary, crizotinib treatment was associated with more grade $3-5$ AEs (53.63\% vs. 37.37\%, RR: 1.43 [1.091.87], $p=0.009)$. The total AEs $(98.74 \%$ vs. $98.16 \%$, RR: 1.01 [0.99-1.03], $p=0.40)$, serious AEs $(29.02 \%$ vs.
24.47\%, RR: 1.12 [0.88-1.44], $p=0.34)$, fatal AEs (3.15\% vs. $2.11 \%$, RR: 1.51 [0.62-3.69], $p=0.37)$, AEs leading to treatment discontinuation (15.77\% vs. $10.79 \%$, RR: 1.37 [0.93-2.02], $p=0.11$ ), AEs leading to dose reduction (21.13\% vs. $19.86 \%$, RR: 1.11 [0.77-1.60], $p=0.57$ ), AEs leading to dose interruption (39.43\% vs. $26.58 \%$, RR: 1.38 $[0.90-2.12], p=0.15)$, and death (0\% vs. $0.53 \%$, RR: 0.20 $[0.01-4.16], p=0.30)$ were comparable between the 2 groups (Table 2).

Subgroup analysis of the total AEs revealed that the crizotinib group reported higher rates of constipation, nausea, diarrhea, vomiting, peripheral edema, dysgeusia, visual impairment, and levels of aspartate aminotransferase (AST) and alanine aminotransferase (ALT) as well as greater decreases in appetite and neutrophil count. However, increased blood bilirubin, bronchitis, and anemia were reported in the alectinib group. Total AEs with an incidence greater than $10 \%$ in the combined crizotinib and alectinib groups are summarized in Table 3 and online supplementary Table S7 and S8.

In the subgroup analysis of grade 3-5 AEs, the crizotinib group exhibited a greater ALT level increase, AST level increase, neutrophil count decrease, electrocardiogram QT prolongation, nausea increase, and vomiting increase. However, anemia was reported in the alectinib group. Grade 3-5 AEs with an incidence greater than $1 \%$ in the combined crizotinib and alectinib groups are summarized in Table 4 and online supplementary Table S9 and S10.

\section{Publication Bias}

The funnel plots for publication bias according to the summary of survival (online suppl. Fig. S6a) and safety 
Table 3. Total adverse events with an incidence of greater than $10 \%$ according to combination of the 2 groups

\begin{tabular}{|c|c|c|c|c|c|c|c|c|c|c|}
\hline \multirow[t]{2}{*}{ Adverse events } & \multirow{2}{*}{$\begin{array}{l}\text { Studies } \\
\text { involved }\end{array}$} & \multicolumn{2}{|l|}{ Crizotinib } & \multicolumn{2}{|l|}{ Alectinib } & \multirow{2}{*}{$\begin{array}{l}\text { Total } \\
\text { incidence, } \\
\%\end{array}$} & \multirow{2}{*}{$\begin{array}{l}\text { Risk } \\
\text { ratio }\end{array}$} & \multirow[t]{2}{*}{$95 \% \mathrm{Cl}$} & \multirow{2}{*}{$\begin{array}{l}P^{2} \\
\%\end{array}$} & \multirow[t]{2}{*}{$p$ value } \\
\hline & & $\begin{array}{l}\text { event/ } \\
\text { total, } n / N\end{array}$ & $\%$ & $\begin{array}{l}\text { event/ } \\
\text { total, } n / N\end{array}$ & $\%$ & & & & & \\
\hline Constipation & 2 & $79 / 166$ & 47.59 & $84 / 228$ & 36.84 & 41.37 & 1.29 & $1.02-1.63$ & 0 & 0.03 \\
\hline Nausea & 3 & $177 / 317$ & 55.84 & $47 / 380$ & 12.37 & 32.14 & 4.15 & $3.13-5.51$ & 40 & $<0.00001$ \\
\hline Diarrhea & 3 & $177 / 317$ & 55.84 & $45 / 380$ & 11.84 & 31.85 & 4.67 & $2.84-7.68$ & 62 & $<0.00001$ \\
\hline ALT increased & 3 & $115 / 317$ & 36.28 & $89 / 380$ & 23.42 & 29.27 & 1.83 & $1.17-2.86$ & 68 & 0.008 \\
\hline Vomiting & 3 & $146 / 317$ & 46.06 & $33 / 380$ & 8.68 & 25.68 & 5 & $3.54-7.07$ & 0 & $<0.00001$ \\
\hline Upper respiratory tract infection & 1 & $20 / 104$ & 19.23 & $28 / 103$ & 27.18 & 23.19 & 0.71 & $0.43-1.17$ & - & 0.18 \\
\hline Nasopharyngitis & 2 & $35 / 166$ & 21.08 & $54 / 228$ & 23.68 & 22.59 & 0.73 & $0.51-1.05$ & 20 & 0.09 \\
\hline Increased blood alkaline phosphatase & 1 & $8 / 62$ & 12.90 & $33 / 125$ & 26.40 & 21.93 & 0.49 & $0.24-0.99$ & - & 0.05 \\
\hline AST increased & 2 & $73 / 255$ & 28.63 & $36 / 255$ & 14.12 & 21.37 & 2.03 & $1.41-2.91$ & 37 & 0.0001 \\
\hline Pyrexia & 1 & $24 / 104$ & 23.08 & $14 / 103$ & 13.59 & 18.36 & 1.7 & $0.93-3.09$ & - & 0.08 \\
\hline Peripheral edema & 3 & $80 / 317$ & 25.24 & $46 / 380$ & 12.11 & 18.08 & 1.93 & $1.39-2.67$ & 0 & $<0.0001$ \\
\hline Dysgeusia & 3 & $94 / 317$ & 29.65 & $25 / 380$ & 6.58 & 17.07 & 4.96 & $1.98-12.41$ & 63 & 0.0006 \\
\hline Creatine phosphokinase increased & 1 & $14 / 104$ & 13.46 & $21 / 103$ & 20.39 & 16.91 & 0.66 & $0.36-1.23$ & - & 0.19 \\
\hline Malaise & 1 & $20 / 104$ & 19.23 & $13 / 103$ & 12.62 & 15.94 & 1.52 & $0.80-2.90$ & - & 0.2 \\
\hline Blood bilirubin increased & 3 & $5 / 317$ & 1.58 & $103 / 380$ & 27.11 & 15.49 & 0.07 & $0.03-0.17$ & 0 & $<0.00001$ \\
\hline Visual impairment & 2 & $75 / 255$ & 29.41 & $4 / 255$ & 1.57 & 15.49 & 16.55 & $1.42-193.51$ & 78 & 0.03 \\
\hline Fatigue & 2 & $35 / 213$ & 16.43 & $40 / 277$ & 14.44 & 15.31 & 1.18 & $0.57-2.41$ & 59 & 0.66 \\
\hline Sinus bradycardia & 2 & $17 / 166$ & 10.24 & $38 / 228$ & 16.67 & 13.96 & 1.51 & $0.16-14.43$ & 77 & 0.72 \\
\hline Decreased appetite & 2 & $44 / 166$ & 26.51 & $9 / 228$ & 3.95 & 13.45 & 7.34 & $3.64-14.77$ & 0 & $<0.00001$ \\
\hline Rash & 3 & $36 / 317$ & 11.36 & $55 / 380$ & 14.47 & 13.06 & 0.75 & $0.40-1.43$ & 52 & 0.39 \\
\hline Bronchitis & 2 & $12 / 166$ & 7.23 & $36 / 228$ & 15.79 & 12.18 & 0.5 & $0.27-0.94$ & 0 & 0.03 \\
\hline Dry skin & 1 & $13 / 104$ & 12.50 & $12 / 103$ & 11.65 & 12.08 & 1.07 & $0.51-2.24$ & - & 0.85 \\
\hline Stomatitis & 1 & $12 / 104$ & 11.54 & $13 / 103$ & 12.62 & 12.08 & 0.91 & $0.44-1.91$ & - & 0.81 \\
\hline Neutrophil count decreased & 2 & $39 / 166$ & 23.49 & $7 / 228$ & 3.07 & 11.68 & 7.57 & $3.37-17.03$ & 0 & $<0.00001$ \\
\hline Blood creatinine increased & 1 & $11 / 104$ & 10.58 & $13 / 103$ & 12.62 & 11.59 & 0.84 & $0.39-1.78$ & - & 0.65 \\
\hline White blood cell count decreased & 1 & $15 / 62$ & 24.19 & $6 / 125$ & 4.80 & 11.23 & 5.04 & $2.06-12.35$ & - & 0.0004 \\
\hline Anemia & 3 & $15 / 317$ & 4.73 & $58 / 380$ & 15.26 & 10.47 & 0.31 & $0.18-0.53$ & 0 & $<0.0001$ \\
\hline
\end{tabular}

AST, aspartate aminotransferase; ALT, alanine aminotransferase; $\mathrm{Cl}$, confidence interval.

(online suppl. Fig. S6b) demonstrated marked evidence of symmetry, indicating an acceptable publication bias. The combined effect size (summary of survival, $Z$ value of $14.56, p<0.00001$; summary of safety, $Z$ value of 4.99 , $p<0.00001)$ indicated that the fail-safe $N$ value was relevant.

\section{Discussion}

ALK-positive lung cancer accounts for $3 \%-7 \%$ of all lung cancer cases and in the past was one of the genetic markers that indicated poor prognosis $[2,22]$. Recently, survival of patients with ALK-positive NSCLC has been greatly improved following the discovery and use of ALK inhibitors $[23,24]$. As the representative first- and second-generation ALK inhibitors, crizotinib and alectinib have been widely used in clinical practice, and their effi- cacy and safety have been assessed [19, 20]. However, whether alectinib exhibits superior antitumor efficacy to crizotinib for the treatment of ALK-positive NSCLC, especially as a first-line treatment, remains controversial [9, 10]. This is the first meta-analysis that focuses on the comparison of crizotinib with alectinib in ALK-positive NSCLC patients based on 3 high-quality RCTs (the ALEX, ALESIA, and J-ALEX clinical trials). In summary, alectinib therapy had better efficacy than crizotinib therapy with regard to OS, PFS, CNS-PFS, DOR, ORR, PR, and grade 3-5 AEs. Additionally, the survival advantages of alectinib over crizotinib increased with the prolongation of survival time. Similar DCR, CR, and total AEs were found in the 2 groups.

Better survival, especially control of CNS metastases, is the primary benefit of alectinib treatment. The advantages of alectinib are significant in both first-line and second-line treatment of ALK-positive NSCLC. Camidge et 
Table 4. Grade 3-5 adverse events with an incidence of greater than $10 \%$ according to combination of the 2 groups

\begin{tabular}{|c|c|c|c|c|c|c|c|c|c|c|}
\hline \multirow[t]{2}{*}{ Grade $3-5$ adverse events } & \multirow{2}{*}{$\begin{array}{l}\text { Studies } \\
\text { involved }\end{array}$} & \multicolumn{2}{|l|}{ Crizotinib } & \multicolumn{2}{|l|}{ Alectinib } & \multirow{2}{*}{$\begin{array}{l}\text { Total } \\
\text { incidence, } \\
\%\end{array}$} & \multirow{2}{*}{$\begin{array}{l}\text { Risk } \\
\text { ratio }\end{array}$} & \multirow[t]{2}{*}{$95 \% \mathrm{Cl}$} & \multirow{2}{*}{$\begin{array}{l}P^{2} \\
\%\end{array}$} & \multirow[t]{2}{*}{$p$ value } \\
\hline & & $\begin{array}{l}\text { event/ } \\
\text { total, } n / N\end{array}$ & $\%$ & $\begin{array}{l}\text { event/ } \\
\text { total, } n / N\end{array}$ & $\%$ & & & & & \\
\hline ALT increased & 3 & $42 / 317$ & 12.93 & $10 / 380$ & 2.63 & 7.32 & 4.66 & $2.36-9.20$ & 0 & $<0.00001$ \\
\hline AST increased & 2 & $21 / 255$ & 8.24 & $9 / 255$ & 3.53 & 5.88 & 2.34 & $1.10-5.00$ & 0 & 0.03 \\
\hline Neutrophil count decreased & 3 & $29 / 317$ & 9.15 & $2 / 380$ & 0.53 & 4.45 & 11.41 & $3.64-25.72$ & 0 & $<0.0001$ \\
\hline Pulmonary embolism & 1 & $8 / 151$ & 5.30 & $2 / 152$ & 1.32 & 3.30 & 3.41 & $0.84-13.82$ & 0 & 0.09 \\
\hline Interstitial lung disease & 2 & $6 / 166$ & 3.61 & $6 / 228$ & 2.63 & 3.05 & 1.6 & $0.17-15.24$ & 66 & 0.68 \\
\hline Creatine phosphokinase increased & 1 & $4 / 151$ & 2.65 & $5 / 152$ & 3.29 & 2.97 & 0.79 & $0.22-2.87$ & - & 0.72 \\
\hline Hepatic function abnormal & 1 & $6 / 104$ & 5.77 & $0 / 103$ & 0.00 & 2.90 & 12.88 & $0.73-225.66$ & - & 0.08 \\
\hline Electrocardiogram QT prolonged & 2 & $12 / 255$ & 4.71 & $2 / 255$ & 0.78 & 2.75 & 4.98 & $1.30-19.06$ & 0 & 0.02 \\
\hline Pneumonia & 1 & $3 / 151$ & 1.99 & $4 / 152$ & 2.63 & 2.31 & 0.75 & $0.17-3.32$ & - & 0.71 \\
\hline Neutropenia & 1 & $6 / 151$ & 3.97 & $0 / 152$ & 0.00 & 1.98 & 13.09 & $0.74-230.25$ & - & 0.08 \\
\hline Anemia & 2 & $1 / 255$ & 0.39 & $9 / 255$ & 3.53 & 1.96 & 0.16 & $0.03-0.88$ & 0 & 0.04 \\
\hline Maculopapular rash & 1 & $1 / 104$ & 0.96 & $3 / 103$ & 2.91 & 1.93 & 0.33 & $0.03-3.12$ & - & 0.33 \\
\hline Hyponatremia & 2 & $6 / 213$ & 2.82 & $3 / 277$ & 1.08 & 1.84 & 2.8 & $0.21-36.84$ & 60 & 0.43 \\
\hline Urinary tract infection & 1 & $1 / 151$ & 0.66 & $4 / 152$ & 2.63 & 1.65 & 0.25 & $0.03-2.23$ & - & 0.21 \\
\hline Increased bilirubin conjugated & 1 & $1 / 62$ & 1.61 & $2 / 125$ & 1.60 & 1.60 & 1.01 & $0.09-10.90$ & - & 0.99 \\
\hline Nausea & 3 & $9 / 317$ & 2.84 & $2 / 380$ & 0.53 & 1.58 & 4.71 & $1.14-19.44$ & 0 & 0.03 \\
\hline Photosensitivity reaction & 1 & $3 / 151$ & 1.99 & $1 / 152$ & 0.66 & 1.32 & 3.02 & $0.32-28.71$ & - & 0.34 \\
\hline Pneumonitis & 1 & $4 / 151$ & 2.65 & $0 / 152$ & 0.00 & 1.32 & 9.06 & $0.49-166.82$ & - & 0.14 \\
\hline Pleural effusion & 1 & 2/151 & 1.32 & $2 / 152$ & 1.32 & 1.32 & 1.01 & $0.14-7.05$ & - & 0.99 \\
\hline Acute kidney injury & 1 & $0 / 151$ & 0.00 & $4 / 152$ & 2.63 & 1.32 & 0.11 & $0.01-2.06$ & - & 0.14 \\
\hline Decreased appetite & 2 & $4 / 166$ & 2.41 & $1 / 228$ & 0.44 & 1.27 & 4.23 & $0.80-22.47$ & 41 & 0.09 \\
\hline White blood cell count decreased & 2 & $5 / 166$ & 3.01 & $0 / 228$ & 0.00 & 1.27 & 7.75 & $0.87-68.81$ & 0 & 0.07 \\
\hline Weight increased & 2 & $1 / 213$ & 0.47 & $5 / 277$ & 1.81 & 1.22 & 0.44 & $0.07-2.65$ & 0 & 0.37 \\
\hline Vomiting & 3 & $7 / 317$ & 2.21 & $0 / 380$ & 0.00 & 1.00 & 10.64 & $1.28-88.57$ & 0 & 0.03 \\
\hline Rash & 3 & $3 / 317$ & 0.95 & $4 / 380$ & 1.05 & 1.00 & 1 & $0.29-3.44$ & 41 & 1 \\
\hline
\end{tabular}

AST, aspartate aminotransferase; ALT, alanine aminotransferase; Cl, confidence interval.

al. [20] analyzed the most recent data of the ALEX study and found that alectinib therapy could greatly prolong the PFS of patients with ALK-positive NSCLC compared with crizotinib therapy (34.8 months vs. 10.9 months, HR: 0.43, 95\% CI: 0.32-0.58). Similar results were confirmed in the ALESIA study [14]. Nishio et al. [13] analyzed the CNS efficacy in the J-ALEX trial and found that alectinib could delay brain metastasis and averted the progression of brain metastases. A study by Gadgeel et al. [19] based on the ALEX trial reported superior CNS activity of alectinib therapy for ALK-positive NSCLC regardless of CNS disease or radiotherapy at baseline. Five reasons may explain the benefits observed with alectinib therapy. First, as a highly selective ALK inhibitor, alectinib targets a significantly different ALK tyrosine kinase domain than does crizotinib, which can overcome the secondary mutation of the kinase domain caused by crizotinib [25]. Second, alectinib can inhibit more ALK mutations (such as EML4-ALK, G1269A, C1156Y,
F1174L, 1151Tin, and L1152R), which were identified in lung cancer tissues of crizotinib-resistant patients [6]. Third, alectinib has a stronger affinity for the ALK tyrosine kinase domain, which can increase the depth of response and prolong the DOR (HR: 0.31 [0.23-0.42], $p<$ $0.00001)$, resulting in a longer PFS [26]. Fourth, alectinib (which is not a substrate of $\mathrm{P}$-glycoprotein) can penetrate into the CNS more effectively than crizotinib and delay brain metastasis [27]. Fifth, a higher frequency of AEs during crizotinib therapy resulted in shorter treatment durations $[11,12,14]$. Subgroup analysis of PFS suggested that being an active smoker, having an ECOG-PS of 2, having postoperative recurrence, and RT-PCR as the ALK testing method might be unfavorable factors for alectinib treatment. However, baseline CNS metastases and previous brain radiation might be favorable factors for alectinib treatment. Additionally, the survival advantages of alectinib over crizotinib increased with the prolongation of survival. Ito et al. [28] and Watanabe et al. 
[29] reported that sequential therapy with crizotinib and alectinib after crizotinib failure could provide a better survival benefit than therapy with alectinib or crizotinib alone in patients with ALK-positive NSCLC. Alectinib was also approved by the FDA for the treatment of metastatic, ALK+ NSCLC following crizotinib therapy [24]. In summary, we believe that alectinib is a better choice for ALK-positive NSCLC first-line treatment or second-line treatment, especially for patients with baseline CNS metastases and/or previous brain radiation.

Relatively good safety is another advantage of treatment with alectinib, although alectinib does have a longer treatment duration. Thirteen AEs with an incidence of $>20 \%$ were reported in the crizotinib group (nausea, diarrhea, constipation, vomiting, increased ALT level, dysgeusia, visual impairment, increased AST level, decreased appetite, peripheral edema, decreased neutrophil count, pyrexia, and nasopharyngitis) compared with $8 \mathrm{AEs}$ in the alectinib group (constipation, upper respiratory tract infection, increased blood bilirubin, increased bilirubin conjugated, increased blood alkaline phosphatase, nasopharyngitis, increased ALT level, and increased creatine phosphokinase). Additionally, 15 grade 3-5 AEs were reported with an incidence greater than $2 \%$ in the crizotinib group (increased ALT level, decreased neutrophil count, increased AST level, abnormal hepatic function, pulmonary embolism, prolonged electrocardiogram QT, neutropenia, interstitial lung disease, decreased white blood cell count, nausea, hyponatremia, increased creatine phosphokinase, pneumonitis, decreased appetite, and vomiting) compared with 9 in the alectinib group (anemia, increased AST level, increased creatine phosphokinase, maculopapular rash, pneumonia, interstitial lung disease, increased ALT level, urinary tract infection, and acute kidney injury). The frequency of AEs was similar to that of a previous report by Seto et al. [8] of the phase 1-2 studies. Hida et al. [12] reported that AEs caused more discontinuations and dose interruptions during crizotinib treatment, which might also decrease the efficacy of the drug. Hou et al. [30] evaluated the safety of all approved ALK inhibitors and suggested that alectinib was the safest ALK inhibitor. In the subgroup analysis, more gastrointestinal disorders, nervous system disorders, eye disorders, and metabolism disorders were found in the crizotinib group. However, the alectinib treatment seemed to cause more AEs related to the urinary system, musculoskeletal and connective tissue, and the respiratory system. In summary, although alectinib is safer than crizotinib for patients with ALK-positive NSCLC, its high frequency of grade 3-5 AEs should be considered during treatment.
The current meta-analysis had several limitations. First, we included only articles written in English, which introduces a language bias. Second, only 3 RCTs were included, which decreases the clinical value of the combined data. Third, like most other meta-analyses, all data in our analysis were extracted from previously published articles, which increases the heterogeneity of data in the merged analysis. Fourth, 532/697 patients were from Asia, which decreases the clinical value in other regions. Fifth, significant heterogeneity was found in the analysis of OSR, CNS-PFSR- $6 \mathrm{~m}$, and DCR, which decreased the quality of these results. Sixth, individual patient data meta-analysis and treatment sequence of crizotinib and alectinib were not conducted due to a lack of data, which might decrease the clinical value of the results. Seventh, the median follow-up time was different among trials, which might increase data heterogeneity between the included studies.

\section{Conclusion}

Alectinib exhibited better OS, PFS, CNS-PFS, and safety than crizotinib; thus, alectinib appears to be superior to crizotinib for the treatment of ALK-positive NSCLC. The survival advantages of alectinib increased with the prolongation of survival time. Baseline CNS metastases and previous brain radiation might be favorable factors for alectinib treatment. Although alectinib is safer than crizotinib, its high frequency of grade 3-5 AEs (37.37\%) should be considered during treatment. Additionally, the existing shortcomings of this meta-analysis require further extensive and high-quality trials to resolve and confirm our conclusions.

\section{Acknowledgments}

The authors thank Professor Jichun Liu, MD (Department of Cardio-Thoracic Surgery, The second affiliated hospital of Nanchang University) for his advice and Professor Xiaoshu Cheng, $\mathrm{MD}, \mathrm{PhD}$ (Department of Cardiology, The second affiliated hospital of Nanchang University) for his data collection.

\section{Statement of Ethics}

Due to the nature of this study, no ethical approval was required. 


\section{Conflict of Interest Statement}

The authors certify that there are no conflicts of interest regarding this manuscript.

\section{Funding Sources}

This study was supported by the National Natural Science Foundation of China (NSFC), Grant No. (81560345), and the Natural Science Foundation of Jiangxi Province (Grant No. 20181BBG78023). The funding had no role in the design and conduct of the study; collection, management, analysis, and interpretation of the data; preparation, review, or approval of the manuscript; and decision to submit the manuscript for publication.

\section{Author Contributions}

Qinghua Zeng had full access to all of the data in the manuscript and takes responsibility for the integrity of the data and the accuracy of the data analysis. Concept and design: all authors. Acquisition, analysis, or interpretation of data: all authors. Drafting of the manuscript: Qinghua Zeng and Lin Zeng. Critical revision of the manuscript for important intellectual content: Qinghua Zeng, Xiquan Zhang, Shan He, Zhiyong Zhou, Luping Xia, and Wenxiong Zhang. Statistical analysis: Qinghua Zeng, Lin Zeng, and Wenxiong Zhang. Supervision: Qinghua Zeng and Wenxiong Zhang.

\section{Data Availability Statement}

The data sets used and/or analyzed during the current study are available from the corresponding author on reasonable request.

\section{References}

1 Siegel RL, Miller KD, Jemal A. Cancer statistics, 2020. CA Cancer J Clin. 2020;70(1):7-30.

2 Dearden S, Stevens J, Wu YL, Blowers D. Mutation incidence and coincidence in non small-cell lung cancer: meta-analyses by ethnicity and histology (mutMap). Ann Oncol. 2013;24:2371-6.

3 Solomon BJ, Mok T, Kim DW, Wu YL, Nakagawa K, Mekhail T, et al. First-line crizotinib versus chemotherapy in ALK-positive lung cancer. N Engl J Med. 2014;371:2167-77.

4 Shaw AT, Kim DW, Nakagawa K, Seto T, Crinó L, Ahn MJ, et al. Crizotinib versus chemotherapy in advanced ALK-positive lung cancer. N Engl J Med. 2013;368:2385-94.

5 Gridelli C, Peters S, Sgambato A, Casaluce F, Adjei AA, Ciardiello F. ALK inhibitors in the treatment of advanced NSCLC. Cancer Treat Rev. 2014;40:300-6.

6 Kodama T, Tsukaguchi T, Yoshida M, Kondoh O, Sakamoto H. Selective ALK inhibitor alectinib with potent antitumor activity in models of crizotinib resistance. Cancer Lett. 2014;351:215-21.

7 Iwama E, Goto Y, Murakami H, Tsumura S, Sakashita H, Mori Y, et al. Survival analysis for patients with ALK rearrangement-positive non-small cell lung cancer and a poor performance status treated with alectinib: updated results of Lung Oncology Group in $\mathrm{Ky}$ ushu 1401. Oncologist. 2020;25:306-e618.

8 Seto T, Kiura K, Nishio M, Nakagawa K, Maemondo $\mathrm{M}$, Inoue $\mathrm{A}$, et al. $\mathrm{CH} 5424802$ (RO5424802) for patients with ALK-rearranged advanced non-small-cell lung cancer (AF-001JP study): a single-arm, open-label, phase 1-2 study. Lancet Oncol. 2013;14:590-
9 Ettinger DS, Wood DE, Aggarwal C, Aisner DL, Akerley W, Bauman JR, et al. NCCN guidelines insights: non-small cell lung cancer, version 1.2020. J Natl Compr Canc Netw. 2019;17(12):1464-72.

10 Planchard D, Popat S, Kerr K, Novello S, Smit EF, Faivre-Finn C, et al. Metastatic non-small cell lung cancer: ESMO Clinical Practice Guidelines for diagnosis, treatment and follow-up. Ann Oncol. 2018;29(Suppl 4):iv192237.

11 Peters S, Camidge DR, Shaw AT, Gadgeel S, Ahn IS, Kim DW, et al. Alectinib versus crizotinib in untreated ALK-positive non-smallcell lung cancer. N Engl J Med. 2017;377:82938.

12 Hida $\mathrm{T}$, Nokihara $\mathrm{H}$, Kondo $\mathrm{M}$, Kim $\mathrm{YH}$, Azuma K, Seto T, et al. Alectinib versus crizotinib in patients with ALK-positive nonsmall-cell lung cancer (J-ALEX): an open-label, randomised phase 3 trial. Lancet. 2017; 390(10089):29-39.

13 Nishio M, Nakagawa K, Mitsudomi T, Yamamoto N, Tanaka T, Kuriki H, et al. Analysis of central nervous system efficacy in the J-ALEX study of alectinib versus crizotinib in ALKpositive non-small-cell lung cancer. Lung Cancer. 2018;121:37-40.

14 Zhou C, Kim SW, Reungwetwattana T, Zhou J, Zhang Y, He J, et al. Alectinib versus crizotinib in untreated Asian patients with anaplastic lymphoma kinase-positive non-smallcell lung cancer (ALESIA): a randomised phase 3 study. Lancet Respir Med. 2019;7(5): 437-46.

15 Bedas A, Peled N, Maimon Rabinovich N, Mishaeli M, Shochat T, Zer A, et al. Efficacy and safety of ALK tyrosine kinase inhibitors in elderly patients with advanced ALK-positive non-small cell lung cancer: findings from the real-life cohort. Oncol Res Treat. 2019; 42(5):275-82.
16 Jadad AR, Moore RA, Carroll D, Jenkinson C, Reynolds DJ, Gavaghan DJ, et al. Assessing the quality of reports of randomized clinical trials: is blinding necessary? Control Clin Trials. 1996;17(1):1-12.

17 Guyatt GH, Oxman AD, Schünemann HJ, Tugwell P, Knottnerus A. GRADE guidelines: a new series of articles in the journal of clinical epidemiology. J Clin Epidemiol. 2011;64(4): $380-2$.

18 Higgins JP, Altman DG, Gøtzsche PC, Jüni P, Moher D, Oxman AD, et al. The Cochrane Collaboration's tool for assessing risk of bias in randomised trials. BMJ. 2011;343:d5928.

19 Gadgeel S, Peters S, Mok T, Shaw AT, Kim DW, Ou SI, et al. Alectinib versus crizotinib in treatment-naive anaplastic lymphoma kinase-positive $(\mathrm{ALK}+)$ non-small-cell lung cancer: CNS efficacy results from the ALEX study. Ann Oncol. 2018;29(11):2214-22.

20 Camidge DR, Dziadziuszko R, Peters S, Mok $T$, Noe J, Nowicka M, et al. Updated efficacy and safety data and impact of the EML4-ALK fusion variant on the efficacy of alectinib in untreated ALK-positive advanced non-small cell lung cancer in the Global Phase III ALEX Study. J Thorac Oncol. 2019;14(7):1233-43.

21 Nakagawa K, Hida T, Nokihara H, Morise M, Azuma K, Kim YH, et al. Final progressionfree survival results from the J-ALEX study of alectinib versus crizotinib in ALK-positive non-small-cell lung cancer. Lung Cancer. 2020;139:195-9.

22 He Y, Gong R, Sun LY, Zhang ZC, Liu XY, Shao $Q$, et al. The percentage of anaplastic lymphoma kinase-positive tumor cells has clinical implications for patients with nonsmall cell lung cancer. Genet Test Mol Biomarkers. 2019;23(8):589-97. 
23 Malik SM, Maher VE, Bijwaard KE, Becker RL, Zhang L, Tang SW, et al. U.S. Food and drug administration approval: crizotinib for treatment of advanced or metastatic nonsmall cell lung cancer that is anaplastic lymphoma kinase positive. Clin Cancer Res. 2014; 20(8):2029-34.

24 Larkins E, Blumenthal GM, Chen H, He K, Agarwal R, Gieser G, et al. FDA approval: alectinib for the treatment of metastatic, ALK-positive non-small cell lung cancer following crizotinib. Clin Cancer Res. 2016; 22(21):5171-6.

25 Karachaliou N, Fernandez Bruno M, Bracht JWP, Rosell R. Profile of alectinib for the treatment of ALK-positive non-small cell lung cancer (NSCLC): patient selection and perspectives. Onco Targets Ther. 2019;12: 4567-75.
26 Novello S, Mazières J, Oh IJ, de Castro J, Migliorino MR, Helland $\AA$, et al. Alectinib versus chemotherapy in crizotinib-pretreated anaplastic lymphoma kinase (ALK)-positive non-small-cell lung cancer: results from the phase III ALUR study. Ann Oncol. 2018; 29(6):1409-16.

27 Kodama T, Hasegawa M, Takanashi K, Sakurai Y, Kondoh O, Sakamoto H. Antitumor activity of the selective ALK inhibitor alectinib in models of intracranial metastases. Cancer Chemother Pharmacol. 2014;74:1023-8.
28 Ito K, Hataji O, Kobayashi H, Fujiwara A, Yoshida M, D'Alessandro-Gabazza CN, et al. Sequential therapy with crizotinib and alectinib in ALK-rearranged non-small cell lung cancer: a Multicenter Retrospective Study. Thorac Oncol. 2017;12(2):390-6.

29 Watanabe S, Hayashi H, Okamoto K, Fujiwara K, Hasegawa Y, Kaneda H, et al. Progression-free and overall survival of patients with ALK rearrangement-positive non-small cell lung cancer treated sequentially with crizotinib and alectinib. Clin Lung Cancer. 2016; 17(6):528-34.

30 Hou H, Sun D, Liu K, Jiang M, Liu D, Zhu J, et al. The safety and serious adverse events of approved ALK inhibitors in malignancies: a meta-analysis. Cancer Manag Res. 2019;11: 4109-18. 